\title{
Automatic 3D Mesh Generation for A Domain with Multiple Materials*
}

\author{
Yongjie Zhang ${ }^{1}$, Thomas J.R. Hughes ${ }^{1}$, and Chandrajit L. Bajaj ${ }^{1,2}$ \\ 1 Institute for Computational Engineering and Sciences, The University of Texas at Austin, \\ United States. \\ 2 Department of Computer Sciences, The University of Texas at Austin, United States.
}

\begin{abstract}
This paper describes an approach to construct unstructured tetrahedral and hexahedral meshes for a domain with multiple materials. We have developed an octree-based isocontouring method to construct unstructured 3D meshes for a single material domain. Based on it, we analyze each material change edge instead of sign change edge to figure out interfaces between two materials, and mesh each material region with conforming boundaries. Two kinds of surfaces, the boundary surface and the interface between two different material regions, are meshed and distinguished. Both material change edges and interior edges are analyzed to construct tetrahedral meshes, and interior grid points are analyzed for hexahedral mesh construction. Finally the edge-contraction and smoothing method is used to improve the quality of tetrahedral meshes, and a combination of pillowing, geometric flow and optimization techniques are used for hexahedral mesh quality improvement. The shrink set is defined automatically as the boundary of each material region. Several application results in different research fields are shown.
\end{abstract}

Key words: Unstructured 3D meshes, multiple materials, conforming boundaries, material change edge, pillowing, geometric flow.

\section{Introduction}

Geometric modelling and mesh generation can not only be used in visualization, more importantly, it can also be used in calculations such as finite element simulations. With the development of finite element analysis in various research fields, especially on the frontier research such as computational medicine and computational biology in recent years, geometric modelling and mesh generation become more and more important. In scanned Computed Tomography (CT) or Magnetic Resonance Imaging (MRI) data, usually there are multiple materials. For example as shown in Figure 1, the MRI brain data has been segmented into about 40 areas, and each area has a specific functionality. Sometimes multiple material regions are considered as the analysis domain and quality meshes for all material regions with conforming boundaries are required. However, it is still very challenging to construct good geometric

${ }^{*}$ http://www.ices.utexas.edu/ jessica/paper/meshmaterial

Email addresses: jessica@ices.utexas.edu (Yongjie Zhang), hughes@ices.utexas.edu (Thomas J.R. Hughes), bajaj@cs.utexas.edu (Chandrajit L. Bajaj) 


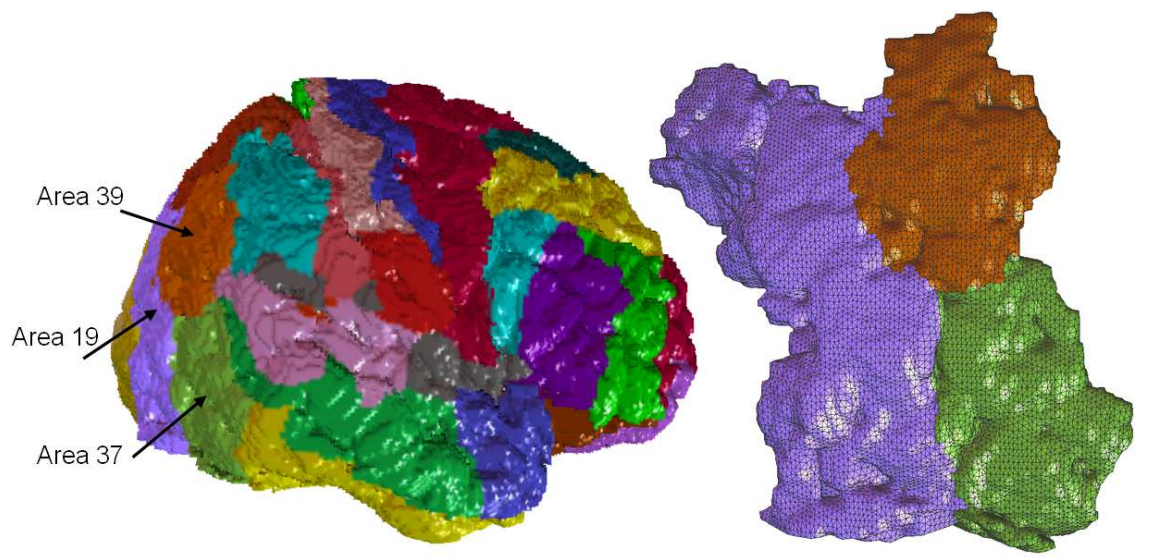

(a)

(b)

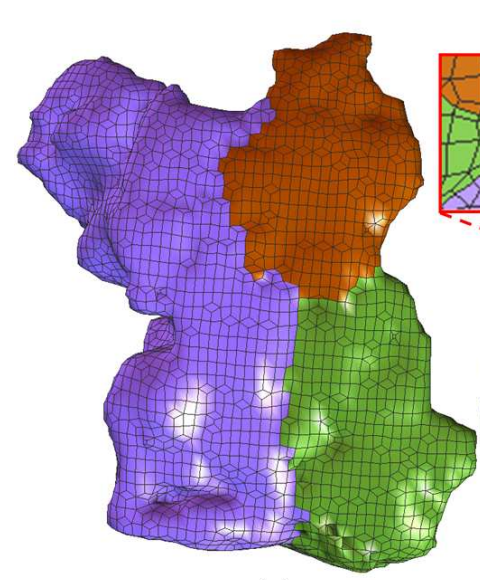

(c)

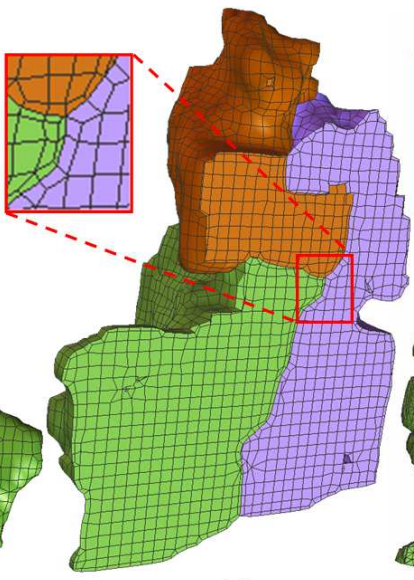

(d)

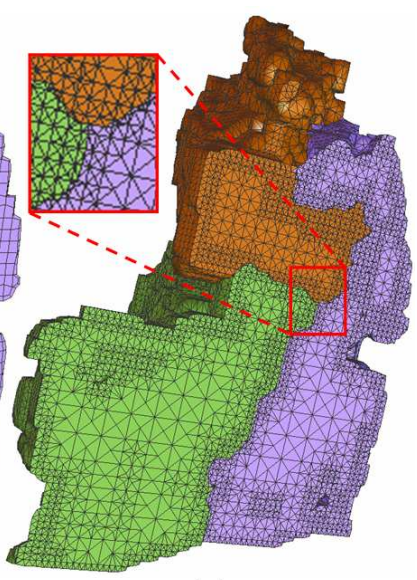

(e)

Fig. 1. Mesh generation for the segmented brodmann brain atlas with about 40 areas or materials. In (b)-(e), only three areas (Area 19, 37, and 39) are shown. (a) - smooth shading of the constructed brain model, each color represents one material; (b) - a triangular mesh; (c) a quadrilateral mesh; (d) - one cross-section of a hexahedral mesh; (e) - one cross-section of a tetrahedral mesh. Red windows show zoom-in pictures.

models for each of these materials. In this paper, we are going to describe an approach to automatically mesh a domain with multiple materials.

We have developed an octree-based isocontouring method [1, 2] to construct adaptive and quality tetrahedral/hexahedral meshes from imaging data with conforming boundaries defined as level sets, but it only works for a domain with a single material. In order to construct 3D meshes for multiple material domains, we analyze material change edge instead of sign change edge to find out all boundaries, including the boundaries of each material domain and the interfaces between two materials. All the boundaries are meshed into triangles or quadrilaterals. Besides material change edge, we also analyze each interior edge for each material domain to construct tetrahedral meshes. Each interior grid point is analyzed for hexahedral mesh construction. 
Mesh adaptivity can be controlled in different ways: by a feature sensitive error function, by regions that users are interested in, by finite element solutions, or by a user-defined error function. The feature sensitive error function measures topology and geometry changes sensitively between isocontours at two neighboring octree levels. Adaptive tetrahedral and hexahedral meshes are generated by balancing the above four criteria and mesh size. Edge contraction and geometric flows [3] are used to improve the quality of tetrahedral meshes. A combination of pillowing, geometric flow, and optimization techniques are chosen for quality improvement of hexahedral meshes. The shrink set is defined in an automatic way, and the pillowing technique guarantees for each element in hexahedral meshes, at most one face lies on the boundary. This provides us a lot of freedom to further improve the aspect ratio, especially for elements along the boundary.

We have applied our meshing method on a segmented human brain data and the rice dwarf virus (RDV) data with multiple materials. Quality tetrahedral and hexahedral meshes are generated automatically with conforming boundaries, and some quantitative statistics such as area and volume for each domain are computed. Our results provide useful information to check the anatomy of the human brain, or identify and understand the RDV structure.

The remainder of this paper is organized as follows: Section 2 talks about related previous work. Section 3 reviews the octree-based unstructured mesh generation techniques we developed. Section 4 discusses detailed algorithm of mesh generation for a domain with multiple materials. Section 5 explains how to improve the mesh quality using various techniques. Section 6 presents some results. Section 7 draws conclusions and outlines planned future work.

\section{Previous Work}

Octree-based Mesh Generation: The octree technique [4, 5], primarily developed in the 1980s, recursively subdivides the cubes containing the geometric model until the desired resolution is reached. Irregular cells are formed along the geometry boundary, and tetrahedra are generated from both the irregular cells on the boundary and the interior regular cells. Unlike Delaunay triangulation and advancing front techniques, the octree technique does not preserve a pre-defined surface mesh. The resulting meshes also change as the orientation of octree cells changes. In order to generate high quality meshes, the maximum octree level difference during recursive subdivision is restricted to be one. Bad elements may be generated along the boundary, therefore quality improvement is necessary after mesh generation.

The grid-based approach generates a fitted 3D grid of structured hex elements on the interior of the volume [6]. In addition to regular interior elements, hex elements are added at the boundaries to fill gaps. The grid-based method is robust, however it tends to generate poor quality elements along the boundary. The resulting meshes are also highly dependent upon the grid orientation, and all elements have similar sizes. A template-based method was developed to refine quad/hex meshes locally [7].

Quality Improvement: Algorithms for mesh improvement can be classified into three categories [8] [9]: local refinement/coarsening by inserting/deleting points, local remeshing by face/edge swapping and mesh smoothing by relocating vertices. Laplacian smoothing generally relocates the vertex position at the average of the nodes connecting to it. Instead of relocating vertices based on a heuristic algorithm, people searched an optimization technique to improve the mesh quality. The optimization algorithm measures the quality of the surrounding elements to a node and attempts to optimize it. The optimization-based smoothing yields better results while it is more expensive. Therefore, a combined Laplacian/optimization-based approach was recommended $[10,11]$, which uses Laplacian smoothing when possible and only uses optimization-based smoothing when necessary. 
Pillowing Techniques: 'Doublet' is formed when two neighboring hexes share two faces, which have an angle of at least 180 degrees. In this situation, it is practically impossible to generate a reasonable jacobian value by relocating vertices. The pillowing technique was developed to remove doublets by refining quad/hex meshes [12]. Pillowing is a sheet insertion operation, which provides a fairly straight-forward method to insert sheets into existing meshes. The speed of the pillowing technique is largely dependent upon the time needed to figure out the shrink set. The number of newly introduced hexahedra equals to the number of quads on the inserted sheet.

We have developed octree-based isocontouring methods to construct tetrahedral and hexahedral meshes from gridded imaging data $[1,2]$. In this paper, we are going to talk about how to extend these methods to automatical tet/hex mesh generation for a domain with multiple materials. In addition, we will also discuss how to automatically define the shrink set and use the pillowing technique to improve the quality of hex meshes.

\section{A Review of Octree-based Isocontouring Method for Mesh Generation}

There are two main isocontouring methods, the primal contouring (or Marching Cubes) and the Dual Contouring. The Marching Cubes algorithm (MC) [13] visits each cell in a volume and performs local triangulation based on the sign configuration of the eight vertices. MC and its variants have three main drawbacks: (1) the resulting mesh is uniform, (2) poor quality elements are generated, and (3) sharp features are not preserved. By using both the position and the normal vectors at each intersection point, the Dual Contouring method [14] generates adaptive isosurfaces with good aspect ratio and preserves sharp features. In this section, we are going to review the Dual Contouring method, and the octree-based algorithms we developed for $3 \mathrm{D}$ mesh generation.

\subsection{Dual Contouring Method}

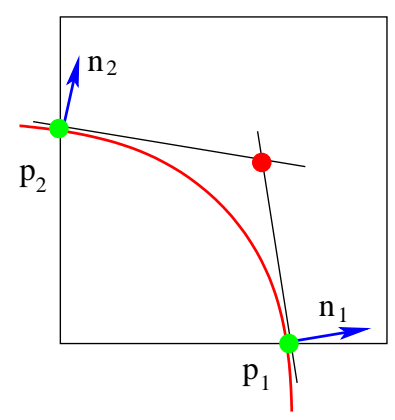

Fig. 2. A minimizer point (the red one) is calculated within an octree cell as the intersection point of two tangent lines. The two green points are two intersections points of the red curve with cell edges. $p_{i}, n_{i}$ are the position and unit normal vectors at a green point respectively.

The octree-based Dual Contouring method [14] analyzes each sign change edge, which is defined as one edge whose two ending points lie on different sides of the isocontour. For each 
octree cell, if it is passed by the isocontour, then a minimizer point is calculated within this cell by minimizing a predefined Quadratic Error Function (QEF) $[15,16]$,

$$
Q E F(x)=\sum_{i}\left(n_{i} \cdot\left(x-p_{i}\right)\right)^{2}
$$

where $p_{i}, n_{i}$ represent the position and unit normal vectors of the intersection point respectively. For example in Figure 2, the red curve is the true curve inside an octree cell, and the two green points are two intersection points of the red curve with cell edges. The calculated minimizer point (the red one) is actually the intersection point of the two tangent lines.

In an uniform case, each sign change edge is shared by four cells, and one minimizer point is calculated for each of them to construct a quadrilateral. In an adaptive case, each sign change is shared by either four cells or three cells, and we obtain a hybrid mesh, including quadrilateral and triangular elements.

\subsection{Tetrahedral Mesh Generation}

We have extended the Dual Contouring method to tetrahedral mesh generation $[1,17]$. Each sign change edge belongs to a boundary cell, which is an octree cell passed through by the isocontour. Interior cell only contains interior edges. In order to tetrahedralize boundary cells, we analyze not only sign change edges but also interior edges. Only interior edges need to be analyzed for interior cell tetrahedralization. Each sign change edge is shared by four or three cells, and we obtain four or three minimizer points. Therefore those minimizers and the interior ending point of this sign change edge construct a pyramid or tetrahedron. For each interior edge, we can also obtain four or three minimizers. Those minimizers and the two ending points of this interior edge construct a diamond or pyramid. A diamond or pyramid can be splitted into four or two tetrahedra. Finally, the edge contraction and smoothing method is used to improve the quality of the resulting meshes.

\subsection{Hexahedral Mesh Generation}

Instead of analyzing edges, we analyze each interior grid point to construct hexahedral meshes from volumetric data [2]. In an uniform case, each grid point is shared by eight octree cells and we can obtain eight minimizers to construct a hexahedron. There are three steps to construct adaptive hexahedral meshes: (1) a starting octree level is selected to construct an uniform hex mesh, (2) templates are used to refine the uniform mesh adaptively without introducing any hanging nodes, (3) an optimization method is used to improve the mesh quality.

These octree-based meshing algorithms we developed are very robust, and they work for complicated geometry and topology. However, they only work for a domain with a single material. In the following section, we are going to talk about how to construct $3 \mathrm{D}$ meshes for a domain with multiple materials.

\section{Mesh Generation for A Domain with Multiple Materials}

\subsection{Problem Description}

Given a geometric domain $\Omega$ consisting of $N$ material regions, denoted as $\Omega_{0}, \Omega_{1}, \ldots$, and $\Omega_{N-1}$, it is obvious that $\cup_{i=1}^{N-1} \Omega_{i}$ is the complementary of $\Omega_{0}$. Suppose $B_{i}$ is the boundary of $\Omega_{i}$, we have $\Omega_{i} \cap \Omega_{j}=B_{i} \cap B_{j}$ when $i \neq j . \cup B_{i}$ may not always be manifold, it can also be nonmanifold curves or surfaces. Figure 3 shows two examples in $2 \mathrm{D}$. There are three materials 
in Figure 3(a) denoted as $\Omega_{0}, \Omega_{1}$ and $\Omega_{2}$, and we can observe that $\cup B_{i}$ are manifold curves. In Figure 3(b), there are four materials, but $\cup B_{i}$ consists of non-manifold curves and a square outer boundary. Non-manifold boundaries $\cup B_{i}$ cannot be represented by isocontours because each data point in a scalar domain can only have one function value. Therefore isocontouring methods do not work for a domain with non-manifold boundaries.

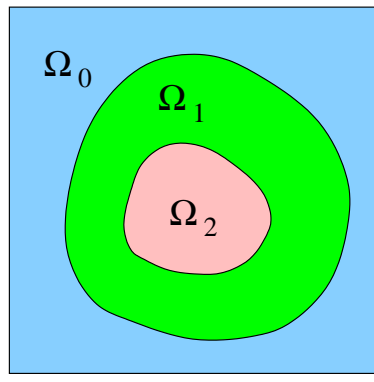

(a)

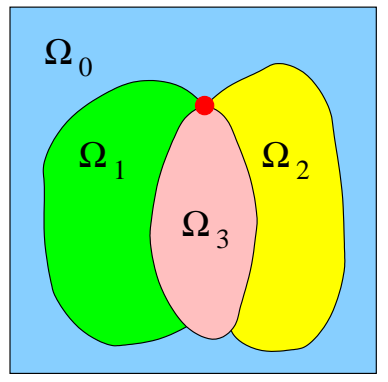

(b)

Fig. 3. A domain with multiple materials. (a) - $\cup B_{i}$ consists of manifold curves; (b) - $\cup B_{i}$ consists of non-manifold curves and a square outer boundary.

One possible way to mesh a domain with non-manifold boundaries is to only consider one material region each time using the method of function modification and isocontouring. After meshes for all the material regions are obtained, we merge them together. However, there are four problems in this method:

1. During the whole process, we have to choose the same octree data structure, especially for interfaces between two different materials. Otherwise the resulting meshes are not conforming to the same boundary.

2. When we mesh each material domain, we detect all boundaries surrounding this material. Part of the boundaries may be shared by more than two materials, for example, the red point in Figure 3(b) is shared by four materials, $\Omega_{0}, \Omega_{1}, \Omega_{2}$, and $\Omega_{3}$. When we process each material, we may obtain four different points to approximate the red one. In another word, the meshes we obtained may not conform to each other around the interface shared by more than two materials.

3. It is difficult to find out the corresponding points on the interface shared by two materials if only position vectors are given.

4. Since we analyze only one material region at one time, we need to process the data $N$ times for a domain with $N$ materials. It is very time consuming.

In this section, we are going to present an approach to automatically detect all boundaries and mesh a domain with multiple materials at the same time. Here are some definitions used in the following algorithm description:

Boundary Cell: A boundary cell is a cell which is passed through by the boundary of a material region.

Interior Cell: An interior cell is a cell which is not passed through by the boundary of any material region.

Material Change Edge: A material change edge is an edge whose two ending points lie in two different material regions. A material change edge must be an edge in a boundary cell. 
Interior Edge: An interior edge is an edge whose two ending points lie inside the same material region. An interior edge is an edge in a boundary cell, or an interior cell.

Interior Grid Point: An interior grid point of one material is a grid point lying inside this material region.

\subsection{Minimizer Point Calculation}

In our octree-based method, only one minimizer point is calculated for each cell, and each octree cell has an unique index. This property provides us a lot of convenience to uniquely index the minimizer point of octree cells without introducing any duplicates.

There are two kinds of octree cells in the analysis domain: interior cells and boundary cells. For each interior cell, we simply choose the center of the cell as the minimizer point. For each boundary cell, we cannot separately calculate the minimizer point using Equation (1) for each material region because different minimizers may be obtained within this cell. For example in Figure 4(a), the red curve is the boundary which is shared by two materials $\Omega_{1}$ and $\Omega_{2}$. The same minimizer (the red point) is obtained when we mesh each material separately. However in Figure 4(b), there are three materials inside the octree cell, $\Omega_{1}, \Omega_{2}$ and $\Omega_{3}$, and the blue point is shared by all those three materials. Three different minimizers (the red points) are obtained for this cell when we mesh each material separately. Therefore instead of mesh each material separately, we include all intersection points (green points) in the QEF and calculate one identical minimizer point within this cell no matter how many materials are contained in it. This guarantees conforming meshes around boundaries.

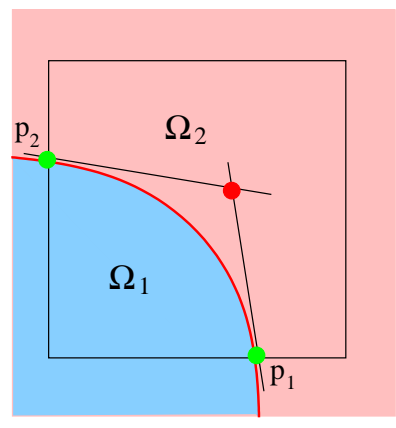

(a)

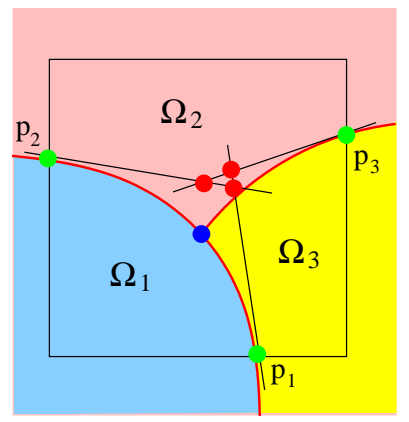

(b)

Fig. 4. Minimizer point calculation within a boundary octree cell. The red curves are boundaries between materials, green points are intersection points of red curves with cell edges, and red points are calculated minimizers. (a) - the boundary is shared by two materials, and the same minimizer is obtained when we mesh each material separately; (b) - the octree cell contains three materials, and the blue point is shared by all the three materials. Three different minimizers are obtained within this cell when we mesh each material separately.

Our meshing algorithm assumes that only one minimizer is generated in a cell. When complicated topology appear in the finest cell, non-manifold surface may be constructed. Schaefer et. al extended the dual contouring method to manifold surface generation [18], but multiple minimizers were introduced within a cell. This cannot be further extended to 3D hexahedral mesh generation. Here we prefer to a heuristic subdivision method. If a cell contains two components of the same material boundary, we recursively subdivide the cell into eight identical 
sub-cells until each sub-cell contains at most one component of the same material boundary. The subdivision method can be easily extended to hex mesh generation, but it may introduce a lot of elements. Fortunately complicated topology rarely happens in a cell, for example in the segmented brain data (Figure 1) and the RDV data (Figure 14), this situation does not exist.

\subsection{D Meshing}

\subsubsection{D Triangulation}

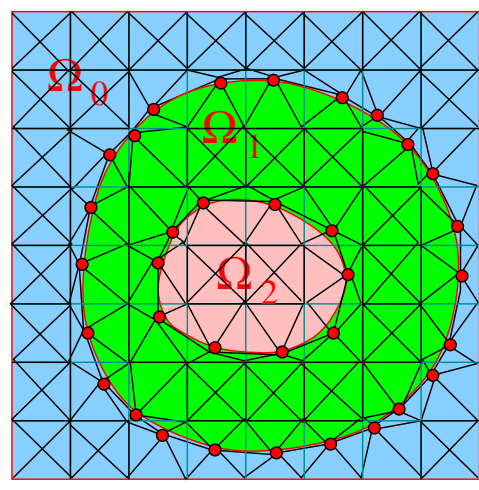

(a)

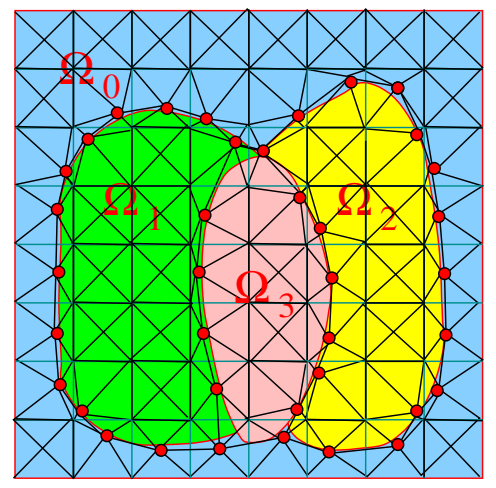

(b)

Fig. 5. 2D triangulation for a domain with multiple materials. (a) - there are three materials, and $\cup B_{i}$ constructs manifold curves; (b) - there are four materials, and $\cup B_{i}$ constructs nonmanifold curves and a square outer boundary.

Material change edges and interior edges are analyzed to construct triangular meshes for each material region as shown in Figure 5:

1. Material change edge: each material change edge is shared by two boundary octree cells in an uniform case, and one minimizer point is calculated by minimizing the quadratic error function defined in Equation (1). We can obtain two minimizer points. The two minimizer points and each ending point of the material change edge construct a triangle. Therefore two triangles are obtained. In an adaptive case, each material change edge is shared by two boundary octree cells too, but the two octree cells may have different sizes. We can also get two minimizer points and construct two triangles.

2. Interior edge: each interior edge is shared by two octree cells. If the octree cell is a boundary cell, then we use Equation (1) to calculate a minimizer point. Otherwise we choose the center of this cell to represent the minimizer point. This interior edge and one minimizer point construct a triangle, therefore two triangles are obtained. In an uniform case, the two ocree cells have the same size, while in an adaptive case, the two ocree cells have different sizes. But we use the same method to construct triangles.

\subsubsection{D Quadrilateral Meshing}

Instead of analyzing edges such as material change edges and interior edges, we analyze each interior grid point to construct a quadrilateral mesh. In an uniform case, each interior grid 
point is shared by four octree cells, and we can calculate four minimizer points to construct a quad. Six templates defined in [2] are used to refine the mesh locally. As shown in Figure 6(a, b), some part of the domain is refined using templates. The mesh quality improvement will be discussed later.

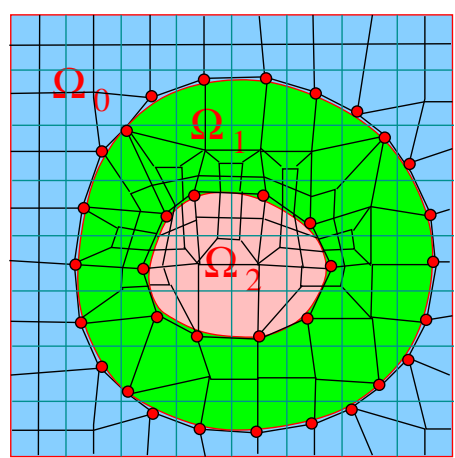

(a)

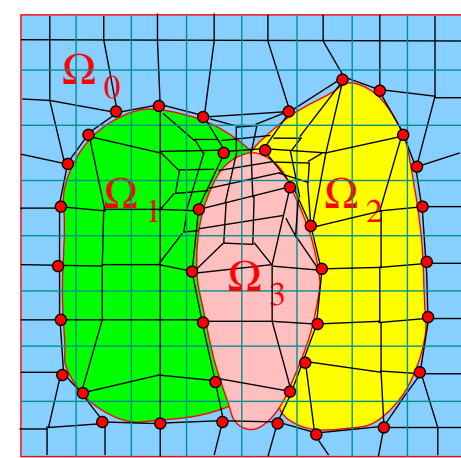

(b)

Fig. 6. 2D quadrilateral meshing for a domain with multiple materials. (a) - there are three materials, and $\cup B_{i}$ constructs manifold curves; (b) - there are four materials, and $\cup B_{i}$ constructs non-manifold curves and a manifold outer boundary.

\subsection{D Meshing}

\subsubsection{Tetrahedral Meshing}

We analyze each edge in the analysis domain, which contains multiple material regions. The edge can be a material change edge or an interior edge. In a 3D uniform case, each edge is shared by four cells. We obtain a total of four minimizers, which construct a quad. The quad and the two ending point of the edge construct two pyramids as shown in Figure 7(a), and each pyramid can be divided into two tetrahedra. In an adaptive case, each edge is shared by four or three octree cells. Therefore we can obtain four or three minimizers, which construct a quad or a triangle. The quad/triangle and the two ending point of the edge construct a diamond or a pyramid as shown in Figure 7(a,b). Finally we split them into tetrahedra.

We have applied our approach on an example with three materials. In Figure 8, a wireframe of the domain is shown in (a), (b) shows the constructed triangular mesh for the surface, and (c) shows one cross-section of the tetrahedral mesh for all these three material regions. It is obvious that they have conforming boundaries.

\subsubsection{Hexahedral Meshing}

For hexahedral mesh generation, we first choose a starting octree level and analyze each interior grid point in an uniform case. In 3D, each edge is shared by eight octree cells, and we can obtain eight minimizer points to construct a hexahedron. An error function is calculated for each octree cell and compared with a threshold to decide the configuration of the minimizer point for this cell. All configurations can be converted into five basic cases defined 


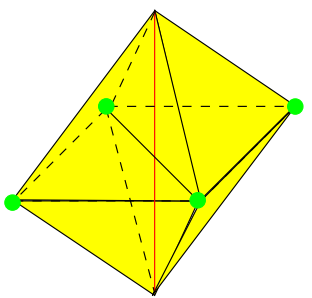

(a)

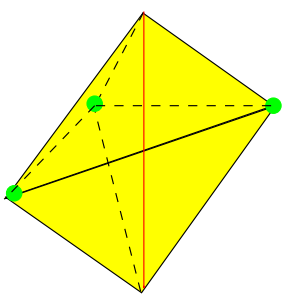

(b)

Fig. 7. Material change edges and interior edges are analyzed in 3D tetrahedralization. (a) - the red edge is shared by four cells, and two pyramids are constructed; (b) - the red edge is shared by three cells, and two tetrahedra are constructed. The green points are minimizer points.

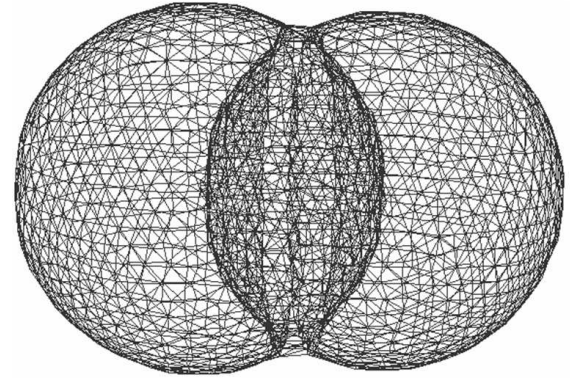

(a)

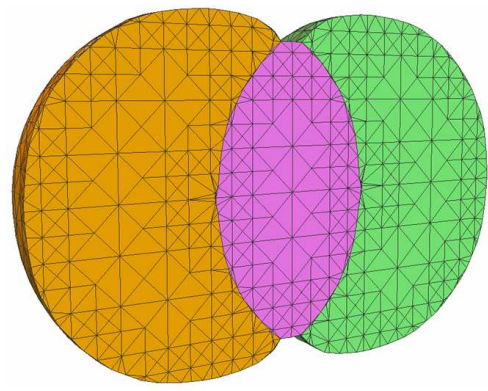

(c)

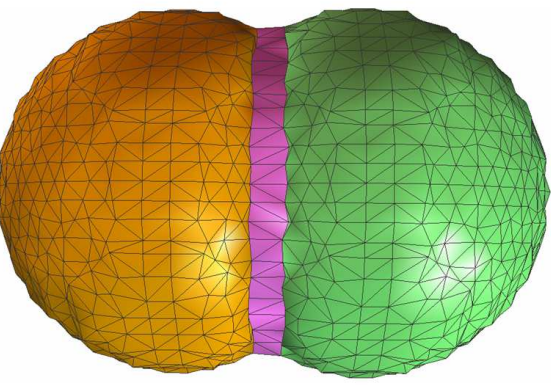

(b)

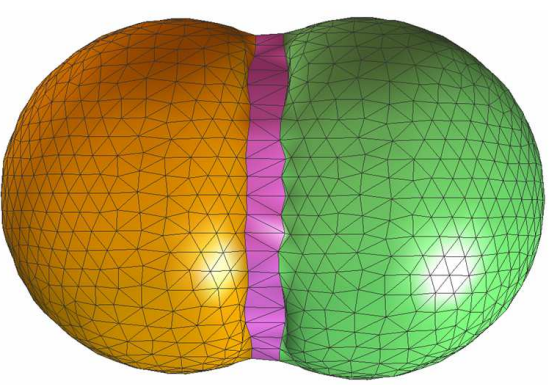

(d)

Fig. 8. Tetrahedral mesh generation for a domain with three materials. (a) - a wireframe visualization; (b) - the constructed triangular surface mesh; (c) - one cross-section of the constructed tetrahedral mesh; (d) - the mesh is improved by edge-contraction and geometric flow.

in $[2,7]$, which are chosen to refine the uniform mesh adaptively without introducing any hanging nodes. The templates satisfy one criteria: in all templates, the refinement around any minimizer points/edges/faces with the same configuration is the same. This criteria guarantees that no hanging node is introduced during the process of mesh refinement. Figure 9(a) shows quadrilateral and hexahedral meshes constructed for a domain with three materials. Figure 9 (c) is one cross-section of the hexahedral mesh. 


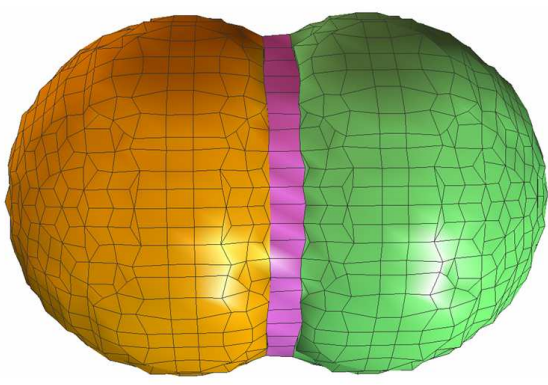

(a)

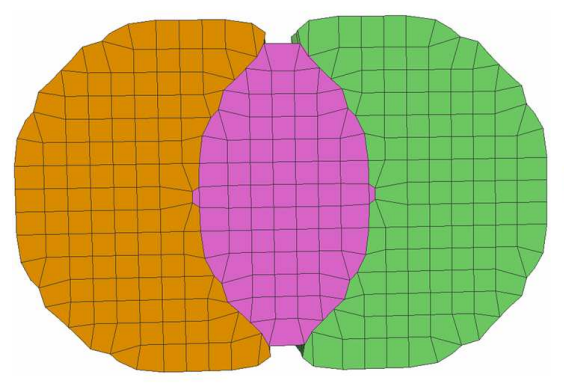

(c)

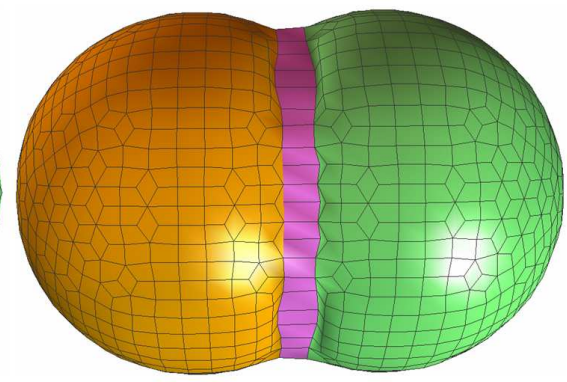

(b)

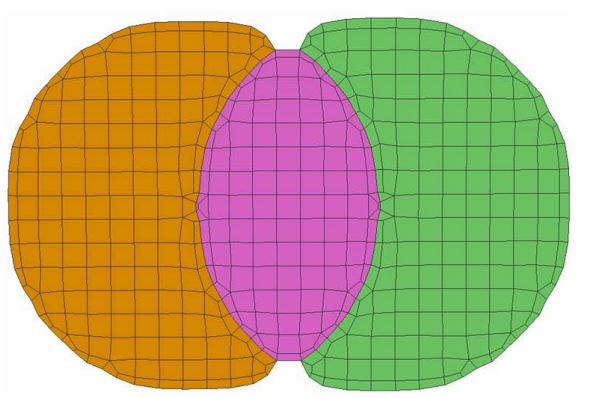

(d)

Fig. 9. Hexahedral mesh construction for a domain with three materials. (a) - surface quad mesh; (b) - surface mesh after quality improvement with geometric flow; (c) - one crosssection of the original hex mesh; (d) - one cross-section of the improved hex mesh.

\subsection{Mesh Adaptivity}

The mesh adaptivity is controlled flexibly by different ways:

1. A feature sensitive function defined in [1] is based on a trilinear function $f^{i}(x, y, z)$ within an octree cell,

$$
F=\sum \frac{\left|f^{i+1}-f^{i}\right|}{\left|\nabla f^{i}\right|}
$$

where

$$
\begin{aligned}
f^{i}(x, y, z) & =f_{000}(1-x)(1-y)(1-z)+f_{001}(1-x)(1-y) z \\
& +f_{010}(1-x) y(1-z)+f_{100} x(1-y)(1-z) \\
& +f_{011}(1-x) y z+f_{101} x(1-y) z \\
& +f_{110} x y(1-z)+f_{111} x y z .
\end{aligned}
$$

$f_{l m n}$ (where $l, m, n=0$ or 1 ) is the function value at a grid point of the octree cell. The feature sensitive error function measures the isocontour difference between two neighboring octree levels, Level $i$ and Level $(i+1)$.

2. Regions where users are interested in. According to various application requirements, location can be included in the error function to control the mesh adaptivity.

3. Finite element solutions. The physical domain is approximated by finite element solutions. We can also use them to efficiently and dynamically control the mesh adaptivity. 
4. User-defined error function. Our algorithm is very flexible, and a user-defined error function can be plugged into the code for the mesh adaptivity control.

\section{Quality Improvement}

Mesh quality is a very important factor influencing the convergence and stability of finite element solvers. In the meshes generated from the above algorithm, most elements have good aspect ratio, especially for interior elements. Some elements lying around the boundaries may have poor aspect ratio, therefore the mesh quality needs to be improved.

\subsection{Tetrahedral Mesh}

First we choose three quality metrics to measure the quality of tetrahedral meshes, then use edge-contraction and geometric flow smoothing to improve it. The three quality measures are: (1) edge-ratio, which is defined as the ratio of the longest edge length over the shortest edge length in a tetrahedron; (2) Joe-Liu parameter $\frac{2^{\frac{4}{3}} \times 3 \times|V|^{\frac{2}{3}}}{\sum_{0 \leq i<j \leq 3}\left|e_{i j}\right|^{2}}$, where $|V|$ denotes the volume, and $e_{i j}$ denotes the edge vectors representing the 6 edges [19]; (3) Minimum volume bound.

Edge-contraction: We detect the element with the worst edge-ratio, and use the edgecontract method to remove it until the worst edge-ratio arrives at a predefined threshold, e.g., 8.5. A special case is shown in Figure 10. When one vertex $P$ is embedded in a triangle $T_{t r i}$ or a tetrahedron $T_{t e t}$, this vertex and each edge of $T_{t r i}$ construct a triangle in $2 \mathrm{D}$, or this vertex and each face of $T_{\text {tet }}$ construct a tetrahedron in 3D. If we contract any edge of $T_{t r i}$ or $T_{t e t}$ before removing the vertex $P$, then we will generate two duplicated and useless elements. This special case needs to be detected, duplicated vertices/elements and useless vertices needs to be removed after edge-contraction.

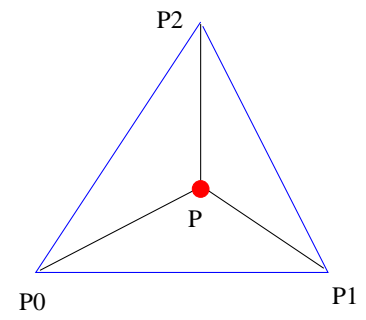

(a)

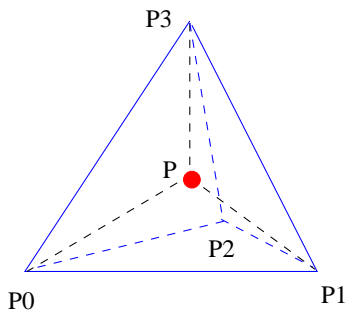

(b)

Fig. 10. A special case for edge-contraction. In (a), a point $P$ is embedded in a triangle $P_{0} P_{1} P_{2}$. $P$ and each edge of the triangle $P_{0} P_{1} P_{2}$ construct a triangle. In (b), a point $P$ is embedded in a tetrahedron $P_{0} P_{1} P_{2} P_{3} . P$ and each face of the tetrahedron $P_{0} P_{1} P_{2} P_{3}$ construct a tetrahedron. When we contract any edge of triangle $P_{0} P_{1} P_{2}$ or tetrahedron $P_{0} P_{1} P_{2} P_{3}$, duplicated and useless elements are generated.

Geometric flow smoothing: There are two kinds of vertices in 3D meshes, boundary vertices and interior vertices. For each boundary vertex, we use geometric flow to denoise the surface and improve the aspect ratio. For interior vertex, we use weighted averaging method to improve the aspect ratio, e.g., volume-weighted averaging. During the smoothing process, the Joe-Liu parameter and minimum volume bound are chosen as the quality metrics. 
Geometric flow or geometric partial differential equations (PDEs) have been intensively used in surface and image processing [20, 21]. Here we choose surface diffusion flow to smooth the surface mesh because it preserves volume. A discretization scheme for the LaplaceBeltrami operator over triangular meshes is given in [21], we do not go to detail here.

The main aim of edge-contraction is to improve the element with the worst edge-ratio for each iteration, however, the edge-contraction method cannot remove slivers, therefore we should couple it with the smoothing scheme. Geometric flow smoothing tends to improve the mesh globally. We repeat running the two steps until a threshold or an optimized state is reached.

Figure 8 shows the difference of the mesh before and after the quality improvement. Figure 8(b) are original meshes, and Figure 8(d) shows the improved mesh. It is obvious that after quality improvement, the surface mesh is more regular and has better aspect ratio.

\subsection{Hexahedral Mesh}
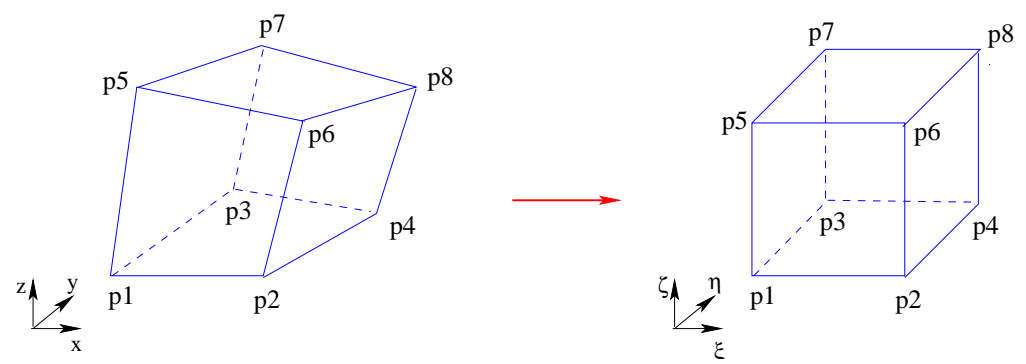

Fig. 11. A hexahedron [p1p2...p8] is mapped into a trilinear parametric volume in terms of $\xi, \eta$, and $\zeta$. The eight basis functions in Equation (4) are attached to the eight vertices of the hexahedron.

Knupp et. al defined the Jacobian matrix of a vertex using its three edge vectors [22, 23], here we choose the definition of the Jacobian matrix in the Finite Element Method [24, 25]. Given a hexahedron with eight vertices as shown in Figure 11, there is a basis function $\phi_{i}$ in terms of $\xi, \eta$ and $\zeta$ attached to each of them in the parametric domain. The eight basis functions are in the following:

$$
\begin{aligned}
\phi_{1} & =(1-\xi) *(1-\eta) *(1-\zeta), \\
\phi_{2} & =\xi *(1-\eta) *(1-\zeta), \\
\phi_{3} & =(1-\xi) * \eta *(1-\zeta), \\
\phi_{4} & =\xi * \eta *(1-\zeta), \\
\phi_{5} & =(1-\xi) *(1-\eta) * \zeta, \\
\phi_{6} & =\xi *(1-\eta) * \zeta, \\
\phi_{7} & =(1-\xi) * \eta * \zeta, \\
\phi_{8} & =\xi * \eta * \zeta .
\end{aligned}
$$

For any point inside this parametric hexahedral element, its coordinates can be calculated as $x=\sum x_{i} * \phi_{i}, y=\sum y_{i} * \phi_{i}$, and $z=\sum z_{i} * \phi_{i}$. The Jacobian matrix is constructed as follows: 


$$
J=\left(\begin{array}{lll}
\frac{\partial x}{\partial \xi} & \frac{\partial x}{\partial \eta} & \frac{\partial x}{\partial \zeta} \\
\frac{\partial y}{\partial \xi} & \frac{\partial y}{\partial \eta} & \frac{\partial y}{\partial \zeta} \\
\frac{\partial z}{\partial \xi} & \frac{\partial z}{\partial \eta} & \frac{\partial z}{\partial \zeta}
\end{array}\right)
$$

The determinant of the Jacobian matrix is called Jacobian, an element is said to be inverted if one of its Jacobians $\leq 0$. We use the Frobenius norm as a matrix norm, $|J|=$ $\left(\operatorname{tr}\left(J^{T} J\right)^{1 / 2}\right)$. The condition number of the Jacobian matrix is defined as $\kappa(J)=|J|\left|J^{-1}\right|$, where $\left|J^{-1}\right|=\frac{|J|}{\operatorname{det}(J)}$. Therefore, the three quality metrics for a vertex $x$ in a hex are defined as $\operatorname{Jacobian}(x)=\operatorname{det}(J), \kappa(x)=\frac{1}{3}\left|J^{-1}\right||J|$, and $\operatorname{Odd} y(x)=\frac{\left(\left|J^{T} J\right|^{2}-\frac{1}{3}|J|^{4}\right)}{\operatorname{det}(J)^{\frac{4}{3}}}$ [26]. A combination of pillowing, geometric flow [3] and optimization techniques is used to improve the quality of hexahedral meshes.

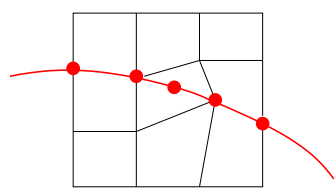

(a)

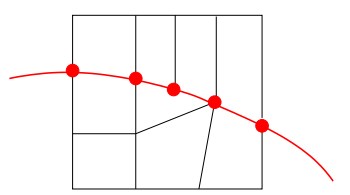

(d)

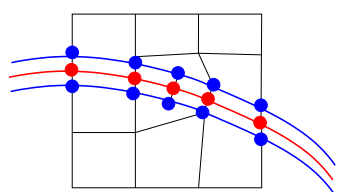

(b)

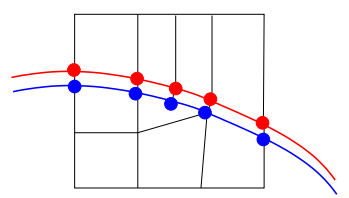

(e)

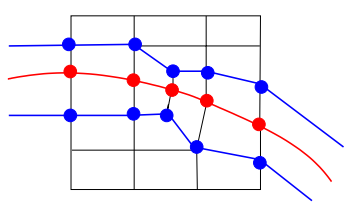

(c)

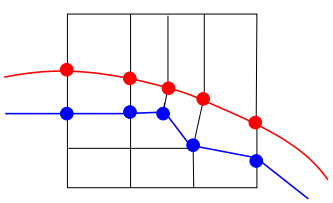

(f)

Fig. 12. The pillowing technique. (a-c) show a 'doublet' and (d-f) show an element whose two faces lie on the boundary, but the two faces are shared by two other elements. $(a, d)$ - the original mesh, the red layer is the boundary shared by two materials; $(b, e)$ - a parallel layer (the blue one) is created for the material with an element whose two faces lie on the boundary. Two layers are created in (b), but only one layer is created in (e); (c, f) - geometric flow is used to smooth the resulting mesh. The red layer is still on the boundary.

Pillowing technique: The pillowing technique was developed to remove 'doublet' as shown in Figure 12(a-c), which is formed when two neighboring hexahedra share two faces. The two faces have an angle of $\geq 180$ degrees, and generally they only appear along the boundaries between two materials. There is another similar situation in our meshes as shown in Figure 12(d-f), two faces of a hexahedron lie on the boundary but they are shared by two other different elements. Since the meshes have to conform to the boundary, it is practically impossible to generate reasonable Jacobian values by relocating vertices. Here we use the pillowing technique to remove the two situations. As shown in Figure 12, first we identify the boundary for each material region, if there is a 'doublet' or an element with two faces on the boundary, then we create a parallel layer/sheet and connect corresponding vertices to construct hexes between the inserted sheet and the identified boundary. The number of newly generated hexes equals to the number of quads on the boundary for each material region. For the boundary shared by two material regions, one or two parallel sheets may be inserted. Finally we use geometric flow to improve the mesh quality. 
The speed of the pillowing technique is largely decided by the time needed to figure out the shrink set, therefore the main challenging problem is how to automatically and efficiently find out where to insert sheets. In our octree data structure, each grid point belongs to one material region. Material change edge is analyzed to construct and detect boundaries, which is shared by two different materials. For example, the two ending points of a material change edge are in two material regions, $\Omega_{1}$ and $\Omega_{2}$. Therefore the constructed boundary is shared by these two materials. In this way, the boundary for each material region is detected, and is defined as the shrink set automatically.

Geometric flow: First boundary vertices and interior vertices are distinguished. For each boundary vertices, there are two kinds movement, one is along the normal direction to remove noise on the boundary, the other one is on the tangent plane to improve the aspect ratio of the mesh. The surface diffusion flow is selected to calculate the movement along the normal direction because the surface diffusion flow preserves the volume. A discretized format of Laplace-Beltrami operator is computed numerically [3]. For each interior vertex, we choose volume-weighted averaging method to relocate it.

Optimization method: After applying pillowing and geometric flow techniques on the meshes, we use the optimization method to further improve the mesh quality. For example, we choose the Jacobian equation as our object function, and use the conjugate gradient method to improve the worst Jacobian value of the mesh. The condition number and the oddy number are also improved at the same time.

We have applied our quality improvement techniques on some hexahedral meshes. In Figure 9, (c) shows one cross-section of the original mesh, and (d) shows the improved mesh. It is obvious that the hexahedral mesh is improved and each element has at most one face lying on the boundary. Figure 13 shows some statistics of quality metrics for the Brodmann brain atlas (Figure 1) and the segmented RDV data (Figure 14).
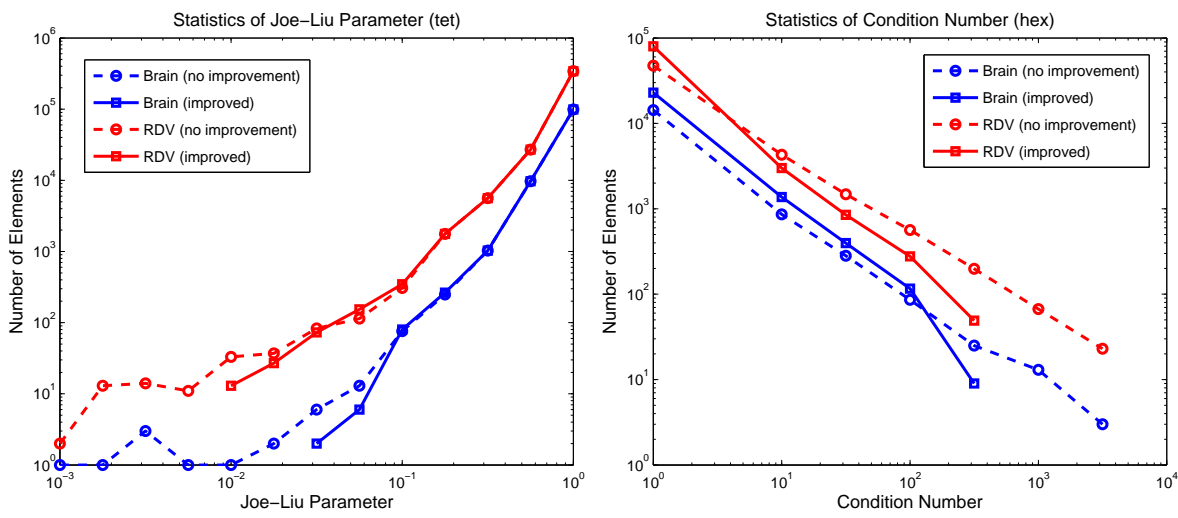

Fig. 13. The histogram of Joe-Liu parameter (left) of tet hexes, and the condition number (right) of hex meshes for the human brain and the RDV data. It is obvious that the mesh quality, especially the worst parameter, is improved 


\section{Results}

In this section, we present applications of our meshing approach to two datasets: the brodmann brain atlas and a segmented rice dwarf virus (RDV) volumetric data.

Brodmann brain atlas: The brodmann brain atlas is a segmented volume map with about 40 different areas or materials, and each area controls a different functionality (http://www.sph. sc.edu/comd/rorden/mricro.html). We apply our meshing algorithms on the atlas to construct meshes for all material regions, and provide some statistic computation such as the surface area and the volume of each region. The results are shown in Figure 1, and three areas are taken to show details of the constructed meshes, including the peristriate area (Area 19, surface area $144.1 \mathrm{~cm}^{2}$ and volume $96.9 \mathrm{~cm}^{3}$ ), the occipitotemporal area (Area 37, surface area $128.9 \mathrm{~cm}^{2}$ and volume $88.7 \mathrm{~cm}^{3}$ ) and the angular area (Area 39, surface area $47.0 \mathrm{~cm}^{2}$ and volume $41.4 \mathrm{~cm}^{3}$ ). The total volume of the brain is $1373.3 \mathrm{~cm}^{3}$ (the normal volume of the human brain is 1300-1500 $\mathrm{cm}^{3}$ ). Some statistics of quality metrics are shown in Figure 13.

Rice dwarf virus: Another main application of our algorithms is on segmented biomolecular data, for example, the cryo-electron magnetic data (cryo-EM) of rice dwarf virus (RDV) with the resolution of 6.8 angstrom. In Figure 14, (a) shows the segmentation result [27], and each color represents four $1 / 3$ unique trimers. Each trimer is further segmented into three P8 monomers as shown in (b-e). Different kinds of meshes are constructed for a trimer which has three materials. Our method provides a convenient approach to visualize the inner structure of RDV. Figure 13 shows some statistics of quality metrics.

\section{Conclusions and Future Work}

We have developed an automatic 3D meshing approach to construct adaptive and quality tetrahedral or hexahedral meshes for a volumetric domain with multiple materials. Boundaries between materials are detected, and each material region is meshed with conforming boundaries. Edge-contraction and geometric flow schemes are used to improve the quality of tetrahedral meshes, while a combination of pillowing, geometric flow and optimization techniques is employed for the quality improvement of hexahedral meshes. We also provide an automatic way to define the shrink set for the pillowing technique.

We have successfully applied our method to three volumetric imaging data, which involve a segmented human brain model and a virus model called rice dwarf virus. As part of the future work, we would like to apply the techniques described here to more applications.

\section{Acknowledgement}

Y. Zhang was partially supported by a J. T. Oden ICES Postdoctoral Fellowship and NSFDDDAS-CNS-054033. C. Bajaj was supported in part by NSF grants EIA-0325550, CNS0540033, and NIH grants P20-RR020647, R01-GM074258, R01-GM073087.

\section{References}

1. Zhang Y., Bajaj C., Sohn B.S. "3D Finite Element Meshing from Imaging Data." The special issue of Computer Methods in Applied Mechanics and Engineering (CMAME) on Unstructured Mesh Generation, vol. 194, no. 48-49, 5083-5106, 2005 


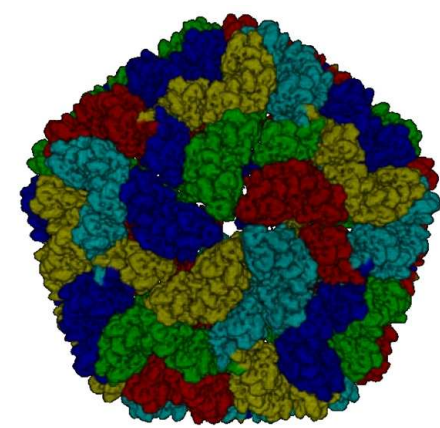

(a)

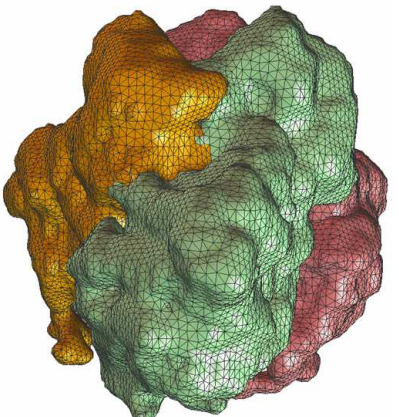

(b)

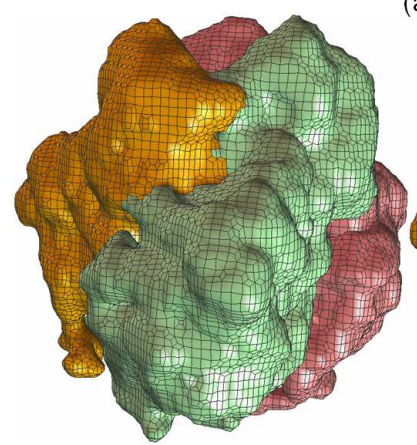

(c)

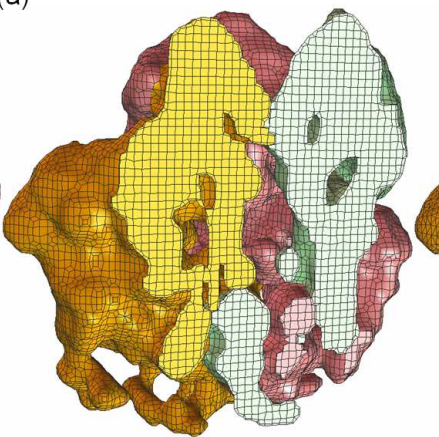

(d)

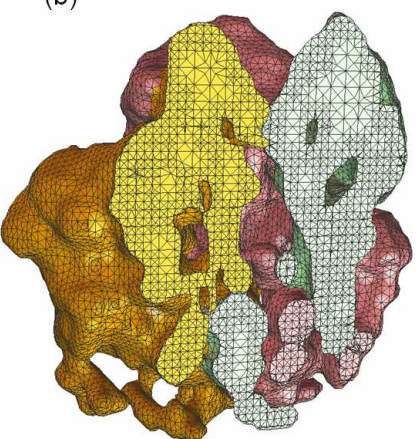

(e)

Fig. 14. Mesh generation for the segmented rice dwarf virus data (RDV). (a) - smooth shading of the segmented RDV model [27], each color represents four 1/3 trimers; (b) - a triangular mesh of one trimer consisting of three monomers; (c) - a quadrilateral mesh of one trimer; (d) - one cross-section of a hexahedral mesh; (e) - one cross-section of a tetrahedral mesh.

2. Zhang Y., Bajaj C. "Adaptive and Quality Quadrilateral/Hexahedral Meshing from Volumetric Data." Computer Methods in Applied Mechanics and Engineering (CMAME), vol. 195, no. 9-12, 942-960, 2006

3. Zhang Y., Bajaj C., Xu G. "Surface Smoothing and Quality Improvement of Quadrilateral/Hexahedral Meshes with Geometric Flow.” Proceedings of 14th International Meshing Roundtable, pp. 449-468. 2005

4. Yerry M.A., Shephard M.S. "Three-Dimensional Mesh Generation by Modified Octree Technique.” International Journal for Numerical Methods in Engineering, vol. 20, 19651990, 1984

5. Shephard M.S., Georges M.K. "Three-Dimensional Mesh Generation by Finite Octree Technique.” International Journal for Numerical Methods in Engineering, vol. 32, 709749, 1991

6. Schneiders R. "A grid-based algorithm for the generation of hexahedral element meshes." Engineering With Computers, vol. 12, 168-177, 1996

7. Schneiders R. "Refining quadrilateral and hexahedral element meshes." 5th International Conference on Grid Generation in Computational Field Simulations, pp. 679-688. 1996

8. Teng S.H., Wong C.W. "Unstructured mesh generation: Theory, practice, and perspectives." International Journal of Computational Geometry and Applications, vol. 10, no. 3, 227-266, 2000

9. Owen S. "A survey of unstructured mesh generation technology." 7th International Meshing Roundtable, pp. 26-28. 1998 
10. Canann S., Tristano J., Staten M. "An approach to combined Laplacian and optimizationbased smoothing for triangular, quadrilateral and quad-dominant meshes." 7th International Meshing Roundtable, pp. 479-494. 1998

11. Freitag L. "On combining Laplacian and optimization-based mesh smoothing techniqes." AMD-Vol. 220 Trends in Unstructured Mesh Generation, pp. 37-43, 1997

12. Mitchell S., Tautges T. "Pillowing Doublets: Refining a Mesh to Ensure That Faces Share at Most One Edge." 4th International Meshing Roundtable, pp. 231-240. 1995

13. Lorensen W.E., Cline H.E. "Marching Cubes: A High Resolution 3D Surface Construction Algorithm." Proceedings of SIGGRAPH, pp. 163-169. 1987

14. Ju T., Losasso F., Schaefer S., Warren J. "Dual Contouring of Hermite Data.” SIGGRAPH, pp. 339-346. 2002

15. Garland M., Heckbert P.S. "Simplifying Surfaces with Color and Texture using Quadric Error Metrics.” IEEE Visualization '98, pp. 263-270. 1998. URL citeseer.nj.nec.com/garland98simplifying.html

16. Hoppe H., DeRose T., Duchamp T., McDonald J., Stuetzle W. "Mesh optimization." SIGGRAPH, pp. 19-26. 1993

17. Zhang Y., Xu G., Bajaj C. "Quality Meshing of Implicit Solvation Models of Biomolecular Structures." The special issue of Computer Aided Geometric Design (CAGD) on Applications of Geometric Modeling in the Life Sciences, vol. 23, no. 6, 510-530, 2006

18. Schaefer S., Ju T., Warren J. "Manifold Dual Contouring.” IEEE Transactions on Visualization and Computer Graphics, accepted, 2007

19. Liu A., Joe B. "Relationship between tetrahedron shape measures." BIT, vol. 34, no. 2, 268-287, 1994

20. Xu G. "Discrete Laplace-Beltrami Operators and Their Convergence." Computer Aided Geometry Design, vol. 21, 767-784, 2004

21. Xu G., Pan Q., Bajaj C. "Discrete Surface Modelling Using Partial Differential Equations." Computer Aided Geometric Design, in press, 2005

22. Knupp P. "Achieving finite element mesh quality via optimization of the jacobian matrix norm and associated quantities. Part II - a framework for volume mesh optimization and the condition number of the jacobian matrix." Int. J. Numer. Meth. Engng, vol. 48, 11651185,2000

23. Kober C., Matthias M. "Hexahedral Mesh Generation for the Simulation of the Human Mandible." 9th International Meshing Roundtable, pp. 423-434. 2000

24. Oden J.T., Carey G.F. "Finite Elements: A Second Course." Prentice Hall, Englewood Cliffs, NJ, 1983

25. Hughes T.J. "The Finite Element Method: Linear Static and Dynamic Finite Element Analysis." Prentice Hall, 1987

26. Oddy A., Goldak J., McDill M., Bibby M. "A distortion metric for isoparametric finite elements." Transactions of CSME, No. 38-CSME-32, Accession No. 2161, 1988

27. Yu Z., Bajaj C. "Automatic Ultra-structure Segmentation of Reconstructed Cryo-EM Maps of Icosahedral Viruses." IEEE Transactions on Image Processing: Special Issue on Molecular and Cellular Bioimaging, vol. 14, no. 9, 1324-1337, 2005 